\title{
Characteristics and risk factors of candidemia in pediatric intensive care unit of a tertiary care children's hospital in Egypt
}

\author{
Moustafa A Hegazi ${ }^{1,2}$, Alaa M Abdelkader ${ }^{2}$, Maysaa E Zaki ${ }^{3}$, Basem S El-Deek ${ }^{4,5}$ \\ ${ }^{1}$ Department of Pediatrics, Faculty of Medicine in Rabigh, King Abdulaziz University, Jeddah, Saudi Arabia \\ ${ }^{2}$ Department of Pediatrics, Mansoura University Children's Hospital, Mansoura, Egypt \\ ${ }^{3}$ Department of Clinical Pathology, Faculty of Medicine, Mansoura University, Mansoura, Egypt \\ ${ }^{4}$ Department of Public Health and Community Medicine, Faculty of Medicine, King Abdulaziz University, Jeddah, \\ Saudi Arabia \\ ${ }^{5}$ Department of Public Health and Community Medicine, Faculty of Medicine, Mansoura University, Mansoura, \\ Egypt
}

\begin{abstract}
Introduction: This study was conducted to determine characteristics of Candida colonization and candidemia in the pediatric intensive care unit (PICU) of a tertiary care children's hospital.

Methodology: Patients between 6 months and 15 years of age consecutively admitted to the PICU of Mansoura University Children's Hospital in Mansoura, Egypt, during one year period, were evaluated for Candida colonization and candidemia. Susceptibility of Candida species isolated from blood to fluconazole and amphotericin B was determined by Etest.

Results: Sixty-six patients without prior fluconazole prophylaxis had 88 episodes of candidemia, representing $19 \%$ of all cases with blood stream infections (BSIs). Candida albicans (CA) and non-albicans Candida (NAC) species accounted for $40 \%$ and $60 \%$ of candidemia episodes respectively. C. parapsilosis, C. tropicalis, and C. glabrata accounted for $25 \%, 17 \%$, and $8 \%$ of NAC candidemias respectively. Fluconazole resistance was detected in $11.4 \%$ and $18.9 \%$ of CA and NAC isolates respectively. Of the fluconazole resistant NAC isolates, four were C. krusei. Amphotericin B resistance was detected in 17\% of NAC isolates. Candida colonization was detected in $78.8 \%$ of patients. Compared to CA candidemia, higher risk for NAC candidemia was associated with age older than 1 year, Candida isolation from endotracheal tube (ETT) and from central venous catheter. Mortality rate was $42.4 \%$, attributable mortality of candidemia was $16.7 \%$. Regression analysis showed that the most significant independent predictors of death were ETT and mechanical ventilation (MV), MV longer than 7 days, and candiduria.

Conclusions: This study presents important epidemiological features of Candida BSIs in a non-neonatal population.
\end{abstract}

Key words: Candida albicans; Candida non-albicans; candidemia risk factors; pediatric intensive care unit

J Infect Dev Ctries 2014; 8(5):624-634. doi:10.3855/jidc.4186

(Received 29 August 2013 - Accepted 12 January 2014)

Copyright $(\underset{c}{ } 2014$ Hegazi et al. This is an open-access article distributed under the Creative Commons Attribution License, which permits unrestricted use, distribution, and reproduction in any medium, provided the original work is properly cited.

\section{Introduction}

Candida bloodstream infections (BSIs) are of particular concern for immunocompromised patients, patients in intensive care settings, patients with central lines, and patients receiving parenteral nutrition and/or broad-spectrum antibiotics for a prolonged period of time [1].

Candidemia is often associated with signs and symptoms of sepsis and fungal infections represent the second highest case-fatality rate (13\%) among all causes of sepsis in children [2].

For many years, Candida albicans (CA) was the principal pathogenic species isolated from blood [3]. However, an increasing role of non-albicans Candida (NAC) species, some of which are intrinsically or potentially resistant to antifungal agents, has been observed. NAC species, particularly C. parapsilosis and $C$. tropicalis, account for almost half of invasive Candida infections in pediatric patients [4,5]. Nonalbicans Candida became more frequent causative agents for invasive fungal infections in the ICU with high transmission of C. parapsilosis [6-8].

As the incidence of NAC BSIs increases, clinicians face challenges regarding empiric treatment of patients with candidemia. In children, most epidemiological studies of candidemia have focused on neonates $[9,10]$. Therefore, risk factors and outcomes in the non-neonatal pediatric population may not be fully estimated. Neonates may have unique risk factors due to their immature immune systems and 
physiological basis for infections. Consequently, data from neonates may not be applicable to the nonneonatal pediatric population [5].

Compared with adults, fewer pediatric patients receive antifungal prophylaxis, so other factors may be associated with the emergence of NAC BSIs in children [11]. The knowledge of local epidemiology and identification of risk factors for NAC BSI in children is important to guide clinicians in the management of these patients [5].

Candida surveillance cultures are very useful tools for identifying and properly treating invasive candidiasis in preterm neonates [10]. However, few data have been reported regarding the colonizationinfection shift outside the NICU where colonization by Candida species was found to be an independent predictor of candidemia in children undergoing treatment for severe sepsis or sepsis shock in PICU [12].

Several limitations were identified in retrospective studies of candidemia where selection bias could be possible due to unavailability of some medical records, incomplete data of the patients with possible lack of information on demographic characteristics, clinical status, cause of death and inability to perform Candida surveillance cultures, preventive interventions and timely adequate treatment for candidemia.

The epidemiology of systemic Candida infections varies in different regions, and frequently, even from one hospital to another within the same region. Consequently, local studies are important in order to obtain relevant epidemiological data and Candida susceptibility profile to anti-fungal drugs in order to assist in the management and treatment of hospitalized patients with Candida infection [13].

No published surveys of candidaemia have ever involved patients in Egypt. Therefore, this surveillance study was conducted to identify the incidence, risk factors, PICU outcome, pattern of Candida colonization, candidemia, and antifungal susceptibilities of isolated organisms in PICU patients of Mansoura University Children's Hospital (MUCH).

\section{Methodology}

\section{Patient selection}

A longitudinal surveillance study was conducted. Infants and children between 6 months and 15 years of age, consecutively admitted to the PICU of Mansoura University Children's Hospital during a period of one year, were recruited in the study. This study was not restricted to specific high-risk groups and included a heterogeneous population of children with a variety of underlying co-morbidities. This study was approved by the local ethics committee of Mansoura Faculty of Medicine, and there was no need for obtaining written informed consent from the parents of all participants.

\section{Exclusion criteria}

Preterm infants with lower than 37 weeks gestation at birth, infants younger than 6 months of age, children older than 15 years, and cases of fungemia not caused by Candida (e.g. cryptococcosis) were excluded from the study. Cases with malignancy and neutropenia with high risk for Candida infection and usually receiving antifungal prophylaxis were also excluded from this study.

Cases of candidemia with concomitant bacteremia were also excluded to avoid the potential contributing effects of bacteremia on the interpretation of resulting data, in order to focus the analysis on cases of isolated candidemia.

\section{Case Definitions}

A case of candidemia was defined as one or more positive blood cultures obtained by a peripheral venipuncture or through an indwelling central venous catheter (CVC) that yielded growth of any Candida species $[7,14]$. Concomitant bacteremia was defined as the isolation of bacterial species from blood within 24 hours since the initial positive fungal blood culture [15].

Apparent clinical resolution was defined as resolution of systemic signs or symptoms (fever, hypotension, tachypnea, etc) that were present on the first day of the onset of candidemia. Microbiological resolution was defined as negative blood cultures for at least two consecutive days without any subsequent positive blood cultures. Both clinical and microbiological resolution was necessary to fulfill the criteria for resolution of that particular episode [5].

Candidemia was considered as catheter related when there was growth of any Candida species from the catheter tip cultured, irrespective of the number of colonies isolated with recovery of the same Candida species grown from simultaneous peripheral venous blood, with accompanying clinical symptoms of infection and no other obvious source of infection [16].

Therapeutic failure was defined as no change or worsening of clinical findings of candidemia while receiving at least five days of antifungal therapy [5].

Mortality was defined as death within 30 days after the onset of candidemia. Candidemia was regarded as the primary cause of death (attributable 
mortality) when a patient died with microbiological and histological or clinical evidence of fungal infection with no other cause identified [17].

\section{Data collection}

The following data were recorded on PICU admission for all patients: age, gender, underlying conditions, reason for admission, referral site (emergency room (ER), hospital ward, or other hospital), length of stay in the referral site and medication used. Emphasis was given to the use of antifungal therapy, and corticosteroids within the preceding two weeks. Data of vascular access (peripheral or central, insertion site, number of replaced CVCs, and duration), as well as the presence of indwelling catheters and endotracheal tubes (ETT) or tracheostomies were also recorded. Pediatric Risk of Mortality score III [18] was calculated for the studied population within the first 24 hours of admission to PICU.

During the PICU stay, variables likely to influence Candida colonization or infection were recorded. Patients were evaluated for length of stay in the PICU, neutropenia, need and duration of mechanical ventilation (MV) and (ETT) intubation, types and duration of administered broad-spectrum antimicrobial agents, use of antifungal therapy, corticosteroids, and total parenteral nutrition (TPN). Outcome (survival for 30 days after PICU discharge or death) was also recorded for each patient.

\section{Diagnosis of Candida colonization and candidemia}

To evaluate the role of Candida colonization in subsequent candidemia, cultures from sites of possible colonization were performed. Oropharyngeal, rectal, and groin skin swabs were taken on admission to PICU, and were repeated once sepsis was suspected and/or candidemia was confirmed. Screening blood cultures for candidemia were taken on admission to PICU. Two additional blood culture samples (48 hours apart) were collected if a patient developed signs of sepsis or catheter associated infection and thereafter as necessary for follow up. Blood cultures from the CVC or peripheral veins and also specimens from relevant sites of suspected infection (eg. bronchial aspirates, urine) were obtained for microbiological investigations including those for fungal infection. Catheter tips and other devices (ETT, urinary catheters) were cultured after removal if a catheterassociated infection was suspected to be responsible for development of sepsis as well as at any other occasion that required replacement of the catheter.
Microbiologic studies and yeast species identification

Different specimens such as blood, urine, skin swabs, nasopharyngeal swabs, rectal samples and catheter samples were collected. Blood samples were subjected to complete microbiologic investigations including aerobic and anaerobic blood cultures, and fungal cultures bottles were incubated in a BACTEC 9050 blood culture system (Becton Dickinson, Franklin Lakes, USA). For other samples, including catheter samples direct culture on Sabouraud dextrose agar (SDA) with chloramphenicol (Oxoid, Basingstoke, UK) was used. After 3 days of incubation at $37^{\circ} \mathrm{C}$ yeast-like fungi were grown on SDA. Yeast species identification was performed using the automated MicroScan system (Siemens Healthcare Diagnostic Inc., USA). Chromogenic tests were based upon detecting the presence of preformed enzymes in unknown organism.

\section{Antifungal susceptibility testing}

Susceptibility of Candida species isolated from blood stream to fluconazole and amphotericin B was tested by the agar-based Etest (Biomerieux, Askim, Sweden) according to the manufacturer's instructions with RPMI 1640 medium supplemented with 2\% glucose. Antifungal susceptibility was tested only for fluconazole and amphotericin B due to availability of their Etest strips as they were the most common antifungals used for treatment in our institute.

Etest MICs were determined after 48 hours of incubation at $35^{\circ} \mathrm{C}$ as the lowest drug concentration at which the zone of complete growth inhibition intersected the scale on the antifungal test strip. The accepted resistance breakpoint for amphotericin B was $\geq 1.0 \mathrm{mg} / \mathrm{L}$. For fluconazole the breakpoints were $\geq 8$ $\mathrm{mg} / \mathrm{L}$ for C. albicans, C. parapsilosis, C. tropicalis, and $\geq 64 \mathrm{mg} / \mathrm{L}$ for $C$. glabrata; no breakpoints have been established for $C$. krusei as the species is a poor target (PT) for therapy with the drug [19]. MIC50 and MIC90 (the MIC at which $50 \%$ and $90 \%$ of the isolates were inhibited) were also calculated.

\section{Statistical analysis}

Statistical analysis was done using statistical package for social science (SPSS). The qualitative data were presented in the form of numbers and percentages. Chi-square was used to compare qualitative data between two groups. Yates correction was used when appropriate. Odds ratios (OR) and 95 $\%$ confidence intervals (CI) were calculated to estimate the risk. Significance was considered when $\mathrm{p}$ value was less than 0.05 . The most significant 
predictors of mortality by univariate analysis were chosen to perform logistic regressions for adjustment of the risk and estimation of the overall predictability of the significant predictors of mortality in this model.

\section{Results}

Incidence of candidemia

During the period of the study, 589 patients between 6 months and 15 years of age were admitted to PICU. BSIs were diagnosed in 347 patients $(58.9 \%)$. Of these, 66 patients $(19 \%)$ had confirmed candidemia and were included in this study. Candida species were the fifth most frequently isolated agent from BSIs in our PICU.

During 26,505 total PICU days of inpatient surveillance, 88 episodes of Candida BSIs were registered. The incidence of candidemia was 3 per 1,000 inpatient-days.

Demographic and clinical characteristics of candidemia patients

The demographics and clinical characteristics of patients with candidemia are summarized in Table 1.

There was no previous use of any antifungal agent for any patient before PICU admission either for prophylaxis or treatment as none of our patients was having malignancy or was neutropenic or was diagnosed with candidemia before PICU admission.

Fifty-two patients $(78.8 \%)$ were colonized with different species of Candida detected either on admission or during PICU stay at the time of diagnosis of candidemia. Candidemia developed in $42(81 \%)$ of colonized patients; 32 of these patients $(76.2 \%)$ were colonized with the same Candida species that caused candidemia. Oropharyngeal $(48 \%)$ and rectal $(41 \%)$ colonization were more common than skin $(32 \%)$ colonization.

Isolated Candida species, sites of isolation and antifungal susceptibility of isolated species

In total, 88 Candida strains were isolated from blood of 66 patients (including isolates in patients with mixed candidemia). CA and NAC species accounted for $40 \%$ and $60 \%$ of the isolates respectively, with mainly C. parapsilosis, C. tropicalis, and C. glabrata causing $25 \%, 17 \%$, and $8 \%$ of NCA BSI respectively. In Table 2 the details of Candida species and the antifungal susceptibility of the isolated strains from blood cultures of the 66 patients admitted to PICU are presented.

Mixed candidal infections with two or more species of CA and NAC isolated in the same blood culture were detected in seven patients and were excluded from the analysis comparing $\mathrm{CA}$ and NAC infection.

Candidaemia was CVC-related in sixteen cases; $C$. albicans, C. parapsilosis, C. tropicalis, C. krusei, and C. glabrata were isolated from two, six, three, two, and three cases respectively. CVC-related candidemia was associated with age older than 1 year $(65 \%)$, CVC duration longer than 15 days $(85 \%)$, associated ETT and MV (78\%), and TPN (44\%). Candida species were also isolated from other sterile sites as urine and ETT in 26 and 29 patients respectively and were of the same species as those isolated from blood in (85\%) of cases. Candida species isolated from ETT included six C. albicans, ten C. parapsilosis, six C. tropicalis, three C. krusei, and four C. glabrata.

Sixty-five isolates $(73.9 \%)$ were susceptible to both fluconazole and amphotericin B. Fluconazole resistance (MIC $\geq 8 \mathrm{mg} / \mathrm{L}$ ) was detected in four $(11.4 \%)$ and ten $(18.9 \%)$ of CA and NAC isolates respectively. Resistance to fluconazole was found in two $C$. parapsilosis, two $C$. tropicalis and two $C$. glabrata isolates. For $C$. krusei no fluconazole breakpoints have been established as the species is a poor target for therapy with the drug [19], but four C. krusei isolates were considered also resistant to fluconazole. MICs were found to be higher against $C$. glabrata and $C$. krusei isolates than against CA isolates, as expected.

Resistance to amphotericin B considered at MIC $\geq$ $1 \mathrm{mg} / \mathrm{L}$ [19] was detected in nine (17\%) of NAC isolates (Table 2).

Risk factors for NAC candidemia (CA versus NAC)

Compared to $\mathrm{CA}$ candidemia, significantly higher risk for NAC candidemia was mainly associated with age older than 1 year (OR: 8.17, CI: 1.99-36.5, p < 0.001 ), isolation of Candida species from CVC (OR: 2.95, CI: $0.5-22.29, \mathrm{p}=0.014)$, and isolation of Candida from ETT (OR: 3.83, CI: 1.07-14.13, p = 0.04). In Table 3 the comparison between risk factors for CA candidemia and risk factors for NAC candidemia is presented. 
Table 1. Demographics and clinical characteristics of patients with candidemia $(\mathrm{N}=66)$.

\begin{tabular}{|c|c|c|}
\hline Character & Number of patients & Percentage $(\%)$ \\
\hline \multicolumn{3}{|c|}{ Age Median (12 months), IQ R (10-40 months) } \\
\hline$<1 \mathrm{y}$ & 41 & 62.1 \\
\hline $1-5 y$ & 13 & 19.7 \\
\hline $5-15 y$ & 12 & 18.2 \\
\hline Male patients & 36 & 54.5 \\
\hline Previous admission in last 2 months & 20 & 30.3 \\
\hline \multicolumn{3}{|l|}{ Admission to PICU from } \\
\hline Inpatient wards & 50 & 75.8 \\
\hline ER & 7 & 10.6 \\
\hline Referral from other hospital & 9 & 13.6 \\
\hline \multicolumn{3}{|c|}{ Underlying chronic disease or co-morbidity } \\
\hline None & 36 & 54.5 \\
\hline Respiratory tract diseases & 10 & 15.2 \\
\hline Neurological diseases & 8 & 12.1 \\
\hline Cardiovascular diseases & 6 & 9.1 \\
\hline Nephropathy & 3 & 4.5 \\
\hline Endocrinopathy (DKA) & 2 & 3.0 \\
\hline Chronic liver disease & 1 & 1.5 \\
\hline
\end{tabular}

Duration of hospitalization before PICU

(Median: 6 days, IQ R:1-14 days)

0 day

$7 \quad 10.6$

$<7$ days

$48 \quad 72.7$

$>7$ days

11

16.7

High PRISM III score $\geq 15$

$29 \quad 43.9$

Broad spectrum antibiotics use before PICU

(Median: 5 days, IQR: 1-12 days)

Broad spectrum antibiotics $>5$ days before PICU

$24 \quad 36.4$

Colonization with Candida

$52-78.4$

Steroids within 15 days before PICU entry

$7-10.6$

Total CVC

$29 \quad 43.9$

$C V C$ related candidemia

$16 \quad 24.2$

Received TBN

$14 \quad 21.2$

ETT intubation and mechanical ventilation 43

(Median: 6 days, IQ R: 1-37 days)

$\begin{array}{llr}\text { Mechanical ventilation }>\text { 7 days } & 20 & 30.3 \\ \text { PICU } \text { stay }>15 \text { days } & 27 & 40.9\end{array}$

(Median: 12 days, IQR: 1-63 days)

Broad spectrum antibiotics in PICU $>15$ days

44

66.7

(Median: 15 days, IQR: 1-39 days)

Outcome

Total mortality

28

42.4

Attributable mortality from candidemia

11

16.7

PICU: pediatric intensive care unit; ER: emergency room; CVC: central venous catheter; ETT: endotracheal tube; IQR: interquartile range 
Table 2. Candida species isolated from blood cultures of 66 patients admitted to PICU (N=88), and antifungal susceptibility.

\begin{tabular}{|c|c|c|c|c|c|c|c|c|}
\hline \multirow{2}{*}{$\begin{array}{c}\text { Isolated Candida } \\
\text { species }\end{array}$} & \multirow{2}{*}{$\begin{array}{c}\text { No. } \\
(\mathrm{N}=88)\end{array}$} & \multirow{2}{*}{$(\%)$} & \multirow{2}{*}{$\begin{array}{c}\text { Antifungal } \\
\text { agent }\end{array}$} & \multicolumn{3}{|c|}{$\mathrm{MIC}(\mathrm{mg} / \mathrm{L})$} & \multicolumn{2}{|c|}{ Resistant species } \\
\hline & & & & Range & MIC50 & MIC90 & No. & $(\%)$ \\
\hline \multirow{2}{*}{ C. albicans } & \multirow{2}{*}{35} & \multirow{2}{*}{40} & Fluconazole & $1.0-64.0$ & 4.0 & 16.0 & 4 & 11.4 \\
\hline & & & Amphotericin B & $0.032-1.0$ & 0.125 & 0.5 & 0 & 0 \\
\hline \multirow{2}{*}{ C. parapsilosis } & \multirow{2}{*}{22} & \multirow{2}{*}{25} & Fluconazole & $1.0-64.0$ & 1.0 & 2.0 & 2 & 9.0 \\
\hline & & & Amphotericin B & $0.023-0.5$ & 0.125 & 0.25 & 1 & 4.5 \\
\hline \multirow{2}{*}{ C. tropicalis } & \multirow{2}{*}{15} & \multirow{2}{*}{17} & Fluconazole & $1.0-64.0$ & 1.0 & 4.0 & 2 & 13.3 \\
\hline & & & Amphotericin B & $0.023-0.5$ & 0.25 & 0.5 & 1 & 6.7 \\
\hline \multirow{2}{*}{ C. glabrata } & \multirow{2}{*}{7} & \multirow{2}{*}{8} & Fluconazole & $1.0-256.0$ & 32 & 128 & 2 & 28.6 \\
\hline & & & Amphotericin B & $0.023-1.0$ & 0.25 & 0.5 & 4 & 57.1 \\
\hline \multirow{2}{*}{ C. krusei } & \multirow{2}{*}{5} & \multirow{2}{*}{6} & Fluconazole & $4.0-256.0$ & 8.0 & 128 & $\mathrm{PT}^{*}$ & $\mathrm{PT}^{*}$ \\
\hline & & & Amphotericin B & $0.064-1.0$ & 0.25 & 0.5 & 3 & 60 \\
\hline \multirow{2}{*}{ C. famata } & \multirow{2}{*}{3} & \multirow{2}{*}{3} & Fluconazole & $1.0-16.0$ & 2.0 & 8.0 & 0 & 0 \\
\hline & & & Amphotericin B & $0.032-1.0$ & 0.125 & 0.5 & 0 & 0 \\
\hline \multirow{2}{*}{ C. lusitaniae } & \multirow{2}{*}{1} & \multirow{2}{*}{1} & Fluconazole & $0125-8.0$ & 1.0 & 8.0 & 0 & 0 \\
\hline & & & Amphotericin B & $0.032-1.0$ & 0.125 & 0.25 & 0 & 0 \\
\hline
\end{tabular}

Breakpoints: fluconazole $\geq 8 \mathrm{mg} / \mathrm{L}$ for C. albicans, C. parapsilosis, C. tropicalis, and $\geq 64 \mathrm{mg} / \mathrm{L}$ for $C$. glabrata; *no breakpoints have been established for $C$. kruse $i$ as the species is a poor target (PT) for therapy with the drug; amphotericin B $\geq 1.0 \mathrm{mg} / \mathrm{L}$ [19].

Table 3. Comparison between risk factors for C. albicans candiemia and non-albicans Candida species candidemia. Mixed candidal infection with two or more species of CA and NAC isolated in the same blood culture was detected in seven patients and were excluded from the study.

\begin{tabular}{|c|c|c|c|c|c|c|}
\hline \multirow{2}{*}{ Risk factor } & \multicolumn{2}{|c|}{$\mathrm{CA}(\mathrm{N}=21)$} & \multicolumn{2}{|c|}{ NAC $(\mathrm{N}=38)$} & \multirow{2}{*}{ OR for NAC (95\% CI) } & \multirow{2}{*}{ *p value } \\
\hline & $\mathbf{N}$ & $\%$ & $\mathbf{N}$ & $\%$ & & \\
\hline \multicolumn{7}{|l|}{ Age } \\
\hline$<$ lyear & $17 / 21$ & 80.9 & $13 / 38$ & 34.2 & $0.12(0.03-0.5)$ & $<0.0011$ \\
\hline$>$ lyear & $4 / 21$ & 19.1 & $25 / 38$ & 65.8 & $8.17(1.99-36.5)$ & $<0.001^{2}$ \\
\hline Previous admission in the last 2 months & $8 / 21$ & 38.1 & $12 / 38$ & 31.6 & $0.75(0.21-2.64)$ & 0.61 \\
\hline \multicolumn{7}{|l|}{ Admission to PICU from } \\
\hline ER & $3 / 21$ & 14.3 & $4 / 38$ & 10.5 & $0.71(0.11-4.56)$ & 0.66 \\
\hline Inpatient wards & $14 / 21$ & 66.7 & $29 / 38$ & 76.3 & $1.62(0.43-6.10)$ & 0.42 \\
\hline Referral from other hospital & $4 / 21$ & 19.1 & $5 / 38$ & 13.2 & $0.64(0.13-3.36)$ & 0.54 \\
\hline High PRISM III score $\geq 15$ & $10 / 21$ & 47.6 & $16 / 38$ & 42.1 & $0.8(0.24-2.67)$ & 0.71 \\
\hline Hospitalization $>7$ days before PICU & $4 / 21$ & 19.1 & $7 / 38$ & 18.4 & $0.96(0.21-4.63)$ & 0.92 \\
\hline PICU stay $>15$ days & $9 / 21$ & 42.9 & $18 / 38$ & 47.4 & $1.2(0.36-4.03)$ & 0.73 \\
\hline Use of antibiotics $>5$ days before PICU & $12 / 21$ & 57.1 & $25 / 38$ & 65.8 & $1.44(0.42-4.95)$ & 0.51 \\
\hline Antibiotic therapy $>15$ days in PICU & $6 / 21$ & 28.6 & $18 / 38$ & 47.4 & $2.25(0.63-8.27)$ & 0.15 \\
\hline Use of 2 or more antibiotics & $15 / 21$ & 71.4 & $26 / 38$ & 68.4 & $0.87(0.23-3.21)$ & 0.81 \\
\hline Colonization with Candida & $16 / 21$ & 76.2 & $29 / 38$ & 76.3 & $1.61(0.45-5.78)$ & 0.40 \\
\hline Steroids within 15 days before PICU entry & $3 / 21$ & 14.3 & $4 / 38$ & 10.5 & $0.71(0.11-4.56)$ & 0.66 \\
\hline CVC related candidemia & $4 / 21$ & 19.1 & $12 / 38$ & 31.6 & $2.31(0.56-10.08)$ & 0.19 \\
\hline Received TBN & $5 / 21$ & 23.8 & $9 / 38$ & 23.7 & $0.99(0.24-4.11)$ & 0.61 \\
\hline ETT intubation and mechanical ventilation & $15 / 21$ & 71.4 & $28 / 38$ & 73.7 & $1.12(0.29-4.28)$ & 0.85 \\
\hline Mechanical ventilation $>7$ days & $4 / 21$ & 19.1 & $16 / 38$ & 42.1 & $3.09(0.76-13.42)$ & 0.07 \\
\hline Urinary catheter and candiduria & $10 / 21$ & 47.6 & $16 / 38$ & 42.1 & $0.8(0.24-2.67)$ & 0.71 \\
\hline Isolation of Candida species from CVC & $2 / 21$ & 9.5 & $9 / 38$ & 23.7 & $2.95(0.5-22.29)$ & $0.014 \stackrel{2}{=}$ \\
\hline Isolation of Candida species from ETT & $6 / 21$ & 28.6 & $23 / 38$ & 60.5 & $3.83(1.07-14.13)$ & $0.04 \stackrel{2}{2}$ \\
\hline
\end{tabular}

${ }^{1}$ higher risk for CA candidemia; ${ }^{2}$ higher risk for NAC candidemia

Data are expressed as numbers and percentages. ${ }^{*}$ Chi-square test was used; OR: Odds ratio; CI: confidence interval; CA: C. albicans; NAC: non-albicans Candida; PICU: pediatric intensive care unit; CVC: central venous catheter; ETT: endotracheal tube 


\section{Treatment of candidemia}

The choice of antifungal was made by the attending physician and was based on the patients' underlying condition and severity of illness. Fourteen patients $(21.2 \%)$ did not receive any antifungal therapy after the first positive Candida culture was reported as seven patients $(10.6 \%)$ died before the results of fungal culture and susceptibility tests were available, four patients $(7.6 \%)$ died within 48 hours of receiving antifungal therapy, and three patients $(4.5 \%)$ had clinician determination of a catheter-related infection (CRI) that resolved after the line was pulled. The treatment of candidemia included removal and replacement of CVC in case of CRI with isolation of the same Candida species from CVC and peripheral blood. This was performed in 11 out of $16(68.75 \%)$ cases of CRI.

The most common initial antifungal used in CA and NAC candidemia was fluconazole with similar use rate in both $\mathrm{CA}$ and NAC candidemia (64\% versus $61.5 \%$ respectively). Liposomal amphotericin B (L-
$\mathrm{AmB}$ ) was more frequently used in NCA compared to CA candidemia (29\% versus 9.3\%). The median duration of therapy was 12 days for liposomal amphotericin B and 15 days for fluconazole. Liposomal amphotericin B was used as second-line therapy, if the patient had failed to respond or developed toxicity to the previous antifungal therapy.

\section{Therapeutic failure}

Among the study population, 52 patients (78.8\%) received antifungal therapy. Fifteen patients had initial therapeutic failure with systemic antifungal treatment; two were associated with CA candidemia and thirteen with NAC candidemia. These fifteen patients were shifted to L-AmB and five cases responded while ten cases did not respond. Ten patients with NAC did not respond to $\mathrm{L}-\mathrm{AmB}$, six of them had $C$. glabrata, and four had $C$. krusei. The $C$. glabrata MICs to L-AmB were $0.125-1.0 \mathrm{mg} / \mathrm{L}$ and the MIC90 was $0.5 \mathrm{mg} / \mathrm{L}$, while the C. krusei MICs were $0.25-1.0 \mathrm{mg} / \mathrm{L}$ and the MIC90 was $0.5 \mathrm{mg} / \mathrm{L}$.

Table 4. Risk factors for mortality in PICU patients with candidemia. Mixed candidal infections with two or more species of CA and NAC isolated in the same blood culture was detected in seven patients and were excluded from the study.

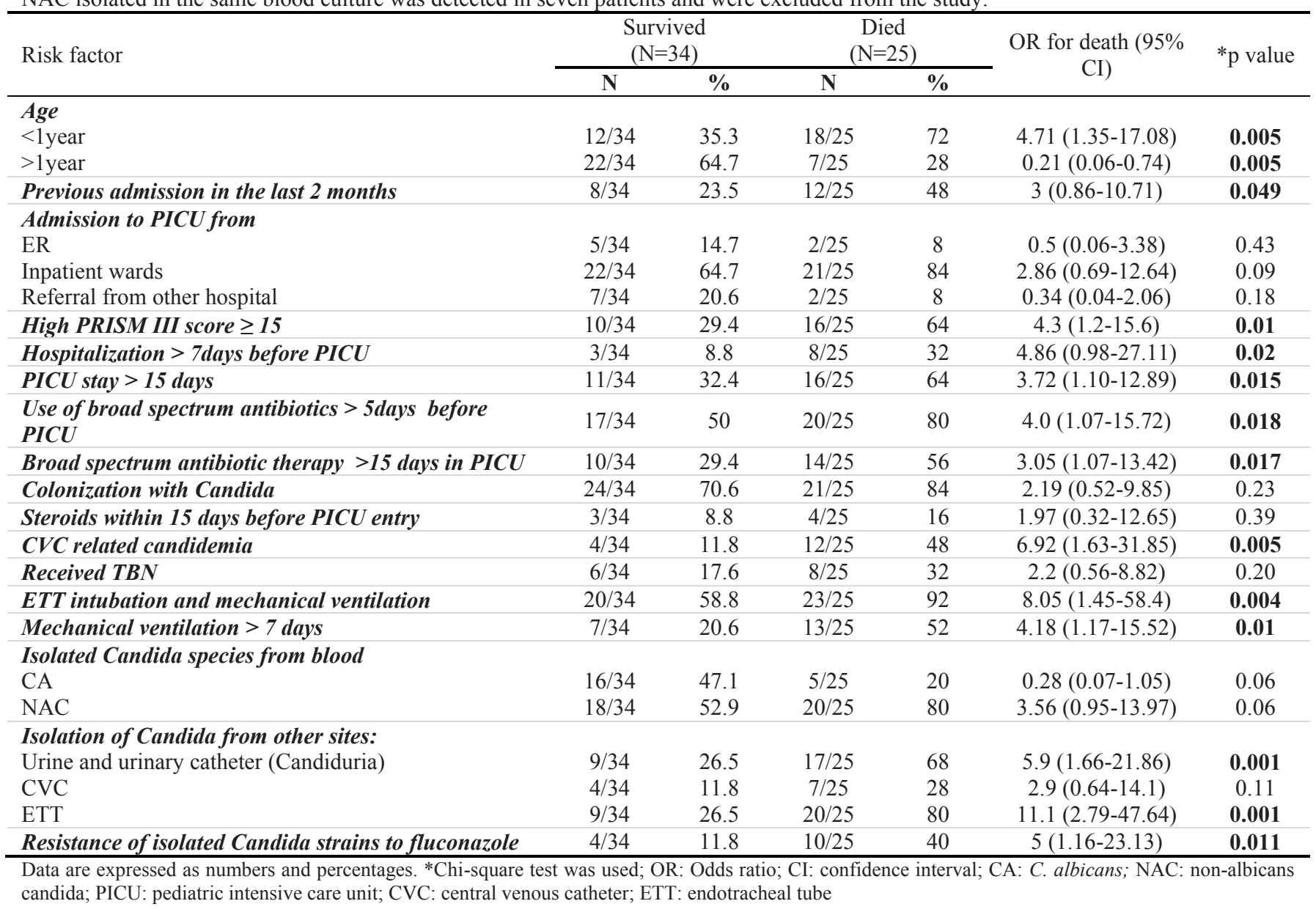




\section{Outcome}

The mortality rate was $42.4 \%$ as 28 cases died, and attributable mortality from candidemia was diagnosed in eleven patients (16.7\%); eight and three deaths were associated with $\mathrm{NAC}$ and $\mathrm{CA}$ BSI respectively. Mortality was higher in NAC candidemia than CA candidemia but this was not statistically significant (OR: 3.56, CI: 0.95-13.967, p =0.06, Table 4). The main isolated NCA species associated with mortality were $C$. glabrata, C. krusei and $C$. tropicalis. Seven out of 10 patients $(70 \%)$ with $C$. glabrata and $C$. krusei who were resistant and did not respond to LAmB, died. In Table 4 the risk factors for mortality in PICU patients with candidemia are shown. The most significant mortality-associated risk factors were age lower than 1 year, previous admission, prolonged hospitalization before PICU, prolonged PICU stay, prolonged use of antibiotics, resistance to fluconazole, and PRISM III score higher or equal to 15.

Multiple stepwise logistic regression showed that the most significant independent predictors of death among total studied cases were ETT and MV (OR: 5.07, CI: 2.96-127.2, $\mathrm{p}=0.032$ ), MV longer than 7 days (OR: 6.21, CI: 1.34-173.5, $\mathrm{p}=0.021$ ), and candiduria (OR: 10.12, CI: 4.63-281.24, $\mathrm{p}=0.002$ ). The overall predictability of this model was $81.4 \%$.

\section{Discussion}

In this study, 66 patients with 88 episodes of confirmed candidemia in non-neonatal age groups were detected in PICU accounting for $11.2 \%$ of total admitted cases, with an overall rate of 3.0 per 1,000 inpatient days. This was a relatively higher incidence rate of candidemia compared to a rate of $10-15 \%$ reported in other studies [20]. Moreover, considering that our study was not restricted to known specific high-risk groups (i.e. neonates, neutropenic, and malignancy patients), this relatively high incidence should be alarming for an emerging serious problem of candidemia at PICU arising as a consequence of management procedures which might have led to the now well-known shift of candidaemia away from its former association with neutropenia into many other patient groups [21].

Non-albicans Candida species in the present study were responsible for $60 \%$ of candidemia cases; with mainly $C$. parapsilosis, $C$. tropicalis, and C. glabrata causing $25 \%, 17 \%$, and $8 \%$ of NAC BSIs respectively (Table 2). These findings are in accordance with the same increasing trend recorded in other studies where NAC BSIs were more prevalent than CA BSIs, with C.parapsilosis, C. tropicalis and C. glabrata being the most common NAC species causing infections in intensive care units $[6,7]$.

However, CA alone was still the most prevalent Candida species responsible for $40 \%$ of Candida BSIs in our PICU, denoting its persistent significant role as the single most common species causing candidemia, a finding which continued to be similarly existing in other studies [5].

We identified $C$. parapsilosis as the second most frequently isolated species after CA, similar to another study in the pediatric age group [22]. The association of C. parapsilosis BSIs and CVC-related candidemia found in this study is consistent with previous reports of the association between $\mathrm{C}$. parapsilosis and $\mathrm{CVC}$ $[21,23,24]$.

Our susceptibility data were similar to those of other studies, and confirmed low levels of antifungal resistance among $C$. albicans isolates but a high level of reduced fluconazole and also reduced amphotericin B susceptibility among $C$. glabrata and C. krusei isolates $[22,25]$. There was considerable agreement between our Etest-MICs results for antifungals including those for amphotericin B, for which clinical breakpoints have not been established yet, and the EUCAST reference method [26,27]. Similarly, Dannaoui et al., found that the level of agreement between Etest and the EUCAST reference method was $75 \%$ for amphotericin $\mathrm{B}$ and ranged from $71 \%$ to $87 \%$ for azoles, even when performed on a routine basis [28].

The increasing prevalence of $C$. krusei and $C$. glabrata should be alarming due to their high MICs and resistance to fluconazole. Surveillance programs continued to show C. krusei as the most fluconazoleresistant Candida species, yet it accounted for only $2.3 \%$ of all isolates [29]. C. glabrata and C. krusei should be considered as serious infectious agents due to their possible inherent resistance to antifungals even without prior exposure to antifungal prophylaxis. Therefore, in the initial management of fungemia caused by $C$. krusei and C. glabrata, empirical use of antifungals should be avoided and targeted treatment should be used instead, after the determination of antifungal susceptibility (AFS).

Widespread fluconazole use can lead to selection for species and strains possessing inherent or acquired azole resistance. These findings have important implications for the management of Candida BSIs, especially given that fluconazole was commonly used for the initial treatment of candidemia in our institute. The negligible incidence of fluconazole resistance among CA isolates may support a practice that 
antifungal susceptibility testing should not be performed routinely for $\mathrm{CA}$, but the increasing prevalence and resistance of NAC to fluconazole should be considered. In fact, a panel of experts of the European Fungal Infection Study Group (EFISG) of the European Society of Clinical Microbiology and Infectious Diseases (ESCMID) considered that AFS must be recommended for patient management for all Candida strains isolated from blood and other deep sites, as reported in recently issued guidelines [19].

In this study and in comparison to CA candidemia, significantly higher risk for NAC candidemia was mainly associated with age older than 1 year, and isolation of NAC species from CVC and ETT. Admission to PICU from inpatient ward, ETT and MV were also associated with candidemia in general, higher NAC than CA, and increased risk for mortality (Tables 1, 3, 4). Few studies have compared the risk factors between patients with CA and NAC BSI in the non-neonatal pediatric population and found no difference in the risk factors between patients with CA and NAC BSI [5,21]. The use of azoles and echinocandins as prophylactic antifungals has been implicated as a major cause for the increase in NAC candidemia [30]. However, prior antifungal exposure was not applicable in this study which included a heterogeneous population of children with a variety of underlying co-morbidities for which antifungal prophylaxis was not indicated.

Candidemia was diagnosed within 24 hours of admission to PICU in $21 \%$ of patients. These patients were coming from inpatient wards, therefore, candidemia in this study was mostly acquired outside the PICU in the absence of antifungal prophylaxis or treatment. Similarly, Hajjeh et al. [21], found that Candida BSIs developed prior to or on the day of admission in $28 \%$ of patients. Thus, considerable proportion of candidemia cases was not identified before PICU admission and consequently missed the opportunity to benefit from earlier diagnosis and administration of systemic antifungal treatment to improve their outcomes. The treating physicians should be aware of the potential risk of candidemia in all hospital settings and not only in intensive care units.

The association of our candidemia cases with age below 1 year, male gender, admission to PICU from inpatient ward, broad spectrum of antibiotics use before PICU, ETT and MV was consistent with the findings of other studies [6,9]. The high association with male gender was also reported by Dutta et al. [5].
Candida colonization shift to candidemia was evident in our PICU patients, and may have been related to their admission in hospital wards and administration of broad spectrum antibiotics for a considerable period of time before PICU admission. This has been also reported in other studies which have pointed to the significant role of Candida colonization as an important risk factor for candidemia even in a non-neonatal population [12]. Moreover, several studies have shown that ICU patients with mucosal Candida colonization, particularly if multifocal, are at higher risk for invasive candidiasis, and that colonization selects a population amenable to antifungal prophylaxis or empirical therapy [31]. Therefore, monitoring for Candida colonization, especially oropharyngeal and rectal colonization in PICU, may offer an opportunity for early detection and management by local antifungals.

In this study, age less than one year was associated with significantly higher CA than NAC candidemia (Table 3). Previous studies showed the same findings, as NAC BSIs were more common than CA BSI in adults and children, while CA BSIs were more common in the infant cohort [1].

Isolation of Candida species from ETT was associated with significantly more NAC species than CA, as well as increased risk for mortality (Tables 3, 4). This finding could suggest disseminated candidemia involving the lungs, although not histopathologically documented. Attention should be directed towards the need for effective treatment of oropharyngeal colonization, as $48 \%$ of our colonized patients developed candidemia which might have resulted from colonization of the ETT tip. More strict precautions and completely aseptic techniques during insertion and standard care of ETT and ventilator circuits should be the same as the standards followed for insertion and subsequent care of CVC.

In this study, the total mortality rate $(42.4 \%)$ from candidemia, the association with NAC species (especially resistant C. glabrata and C. krusei) and the increased risk for mortality in infants younger than 1 year, were similar to mortality rates from candidemia reported in other studies where crude mortality rates for candidemia ranged between $43 \%$ to $54 \%$ in infants and $11 \%$ to $26 \%$ in children, with the highest rates reported with $C$. glabrata and $C$. krusei $[3,11,32,33]$. However, in this study, the attributable mortality from candidemia of $16.7 \%$ was relatively lower than that reported by other pediatric centers [3,32]. This could be related to the exclusion of neonates, anticipation of candidemia in our relatively low risk PICU patients 
(without neutropenia or malignancy), and early institution of antifungal treatment and removal of CVC in cases of CRI.

In this study, there was higher mortality from NAC candidemia than CA candidemia but this was not statistically significant ( $p=0.06$, Table 4$)$. Other studies found that children with NAC BSIs were approximately twice more likely to die than children with CA BSIs [1]. In addition, significantly higher risk for mortality was related to many variables regarded as strong markers for severity of illness (Table 4). These seemed to be logical and have been reported in other studies $[3,15]$. In cases of critical illness it is expected that there will be prolonged hospitalization, prolonged antibiotic use, longer duration for $\mathrm{CVC}$ and device placement as ETT and urinary catheters, leading to an anticipated higher mortality. Our multiple logistic regression analysis showed in fact, that the most significant predictors of death among our total studied cases were ETT intubation and MV, MV longer than 7days, and candiduria. In this study, candiduria may be considered as an indirect evidence of dissemination.

To the best of our knowledge, this surveillance study is the first study which thoroughly characterized candidaemia exclusively in the non-neonatal pediatric population, not belonging to specific groups such as neutropenic or oncology patients, in Egypt. These important findings can guide future studies and preventive measures for candiemia at our PICU as well as in other facilities with similar conditions.

\section{Conclusions}

The high incidence of candidaemia and the increasing emergence of NAC species without prior fluconazole exposure in our PICU, relative to other population-based studies, and the high level of reduced fluconazole and amphotericin B susceptibility of $C$. glabrata and $C$. krusei isolates warrant continued future surveillance of invasive Candida infections.

Candidemia should be anticipated in a considerable subset of non-neonatal population which may not be qualified for fluconazole prophylaxis and in whom preventive measures including antifungal prophylaxis should be initiated. Monitoring for Candida colonization and surveillance for candidemia in PICU may be worthy and cost-effective allowing early detection, prevention, and treatment of critically ill patients to decrease mortality and costs of prolonged PICU stay.

The controversial role of fluconazole prophylaxis in the PICU and the fear from the emergence of fluconazole resistant NAC should emphasize the extremely important role of basic hygiene measures that should never be underestimated.

More effective control and strict treatment protocols could be beneficial to patients with well known risk factors for candidemia as presence of CVC, ETT, prolonged broad-spectrum antibiotics use, prolonged hospitalization inside inpatient wards and Candida colonization, as those identified also in our PICU patients.

\section{References}

1. Moran C, Grussemeyer CA, Spalding JR, Benjamin DK Jr, Reed SD (2009) Candida albicans and non-albicans bloodstream infections in adult and pediatric patients: comparison of mortality and costs. Pediatr Infect Dis J 28: 433-435.

2. Watson RS, Carcillo JA, Linde-Zwirble WT, Clermont G, Lidicker J, Angus DC (2003) The epidemiology of severe sepsis in children in the United States. Am J Respir Crit Care Med 167: 695-701.

3. Pappas PG, Rex JH, Lee J, Hamill RJ, Larsen RA, Powderly W, Kauffman CA, Hyslop N, Mangino JE, Chapman S, Horowitz HW, Edwards JE, Dismukes WE (2003) A prospective observational study of candidemia: epidemiology, therapy, and influences on mortality in hospitalized adult and pediatric patients. Clin Infect Dis 37: 634-643.

4. Filioti J, Spiroglou K, Panteliadis CP, Roilides E (2007) Invasive candidiasis in pediatric intensive care patients: epidemiology, risk factors, management, and outcome. Intensive Care Med 33: 1272-1283.

5. Dutta A, Palazzi DL (2011) Candida non-albicans versus Candida albicans fungemia in the non-neonatal pediatric population. Pediatr Infect Dis J 30: 664-668.

6. Kuzucu C, Durmaz R, Otlu B, Aktas E, Gulcan H, Cizmeci Z (2008) Species distribution, antifungal susceptibility and clonal relatedness of Candida isolates from patients in neonatal and pediatric intensive care units at a medical center in Turkey. New Microbiol 31: 401-408.

7. Neu N, Malik M, Lunding A, Whittier S, Alba L, Kubin C, Saiman L (2009). Epidemiology of candidemia at a Children's hospital, 2002 to 2006. Pediatr Infect Dis J 28: 806-809.

8. Singhi S, Deep A (2009) Invasive candidiasis in pediatric intensive care units. Indian J Pediatr 76: 1033-1044.

9. Roilides E, Farmaki E, Evdoridou J. Dotis J, Hatziioannidis E, Tsivitanidou M (2004) Neonatal candidiasis: analysis of epidemiology, drug susceptibility, and molecular typing of causative isolates. Eur J Clin Microbiol Infect Dis 23 745750 .

10. Vendettuoli V, Tana M, Tirone C, Posteraro B, La Sorda M, Fadda G (2008) The role of Candida surveillance cultures for identification of a preterm subpopulation at highest risk for invasive fungal infection. Pediatr Infect Dis J 27: 1114-1116.

11. Zaoutis TE, Coffin SE, Chu JH, Heydon K, Zhao H, Greves HM, Walsh TJ (2005) Risk factors for mortality in children with candidemia. Pediatr Infect Dis J 24: 736 -739. 
12. Singhi S, Rao DS, Chakrabarti A (2008) Candida colonization and candidemia in a pediatric intensive care unit. Pediatr Crit Care Med 9: 91-95.

13. Aquino VR, Lunardi LW, Goldani LZ, Barth AL (2005) Prevalence, susceptibility profile for fluconazole and risk factors for candidemia in a tertiary care hospital in southern Brazil. Braz J Infect Dis 9: 411-418.

14. Lagrou K, Verhaegen J, Peetermans WE, De Rijdt T, Maertens J, Van Wijngaerden E (2007) Fungemia at a tertiary care hospital: incidence, therapy, and distribution and antifungal susceptibility of causative species. Eur J Clin Microbiol Infect Dis 26: 541-547.

15. Celebi S, Hacimustafaoglu M, Ozdemir O and Ozkaya G (2007) Nosocomial candidaemia in children: results of a 9year study. Mycoses 51: 248-257.

16. Subha Rao SD, Joseph MP, Lavi R, Macaden R (2005) Infections related to vascular catheters in a Pediatric Intensive Care Unit. Indian Pediatrics 42: 667-672.

17. Viudes A, Peman J, Canton E, Ubeda P, Lopez-Ribot JL, Gobernado M (2002) Candidemia at a tertiary-care hospital: epidemiology, treatment, clinical outcome and risk factors for death. Eur J Clin Microbiol Infect Dis 21: 767-774.

18. Pollack MM, Patel KM, Ruttimann UE (1996) PRISM III: An updated pediatric risk of mortality score. Crit Care Med 24, 743-752.

19. Cuenca-Estrella M, Verweij PE, Arendrup MC, ArikanAkdagli S, Bille J, Donnelly JP, Jensen HE, Lass-Flörl C, Richardson MD, Akova M, Bassetti M, Calandra T, Castagnola E, Cornely OA, Garbino J, Groll AH, Herbrecht R, Hope WW, Kullberg BJ, Lortholary O, Meersseman W, Petrikkos G, Roilides E, Viscoli C, Ullmann AJ; ESCMID Fungal Infection Study Group. (2012) ESCMID Fungal Infection Study Group: ESCMID guideline for the diagnosis and management of Candida diseases 2012: diagnostic procedures. Clin Microbiol Infect 18: 9-18.

20. Falagas ME, Apostolou KE, Pappas VD (2006) Attributable mortality of candidemia: a systematic review of matched cohort and case-control studies. Eur J Clin Microbiol Infect Dis 25: 419-425.

21. Hajjeh RA, Sofair AN, Harrison LH, Lyon GM, ArthingtonSkaggs BA, Mirza SA (2004) Incidence of bloodstream infections due to Candida species and in vitro susceptibilities of isolates collected from 1998 to 2000 in a population-based active surveillance program. J Clin Microbiol 42: 1519-1527.

22. Pfaller MA, Diekema DJ, Jones RN, Messer SA, Hollis RJ (2002) Trends in antifungal susceptibility of Candida spp. isolated from pediatric and adult patients with bloodstream infections: SENTRY Antimicrobial Surveillance Program, 1997 to 2000. J Clin Microbiol 40: 852-856.

23. Stamos JK, Rowley AH (1995) Candidemia in a pediatric population. Clin Infect Dis 20: 571-575.

24. Barchiesi F, Caggiano G, Falconi DF, Montagna MT, Barbuti S, Scalise G (2004) Outbreak of fungemia due to Candida parapsilosis in a pediatric oncology unit. Diagn Microbiol Infect Dis 49: 269-271.

25. Odds FC, Hanson MF, Davidson AD, Jacobsen MD, Wright P, Whyte JA, Gow NA, Jones BL. (2007). One year prospective survey of Candida bloodstream infections in Scotland. J Med Microbiol 56: 1066-1075.

26. The European Committee on Antimicrobial Susceptibility Testing-Subcommittee on Antifungal Susceptibility Testing: Amphotericin B: Rationale for the EUCAST clinical breakpoints, version 1.0, 9th November 2010. Available: http://www.eucast.org. Accessed 15/12/2012.

27. Lass-Flörl C, Arendrup MC, Rodriguez-Tudela JL, CuencaEstrella M, Donnelly P, Hope W (2011) EUCAST technical note on amphotericin B. Clin Microbiol Infect 17: E27-9.

28. Dannaoui E, Paugam A, Develoux M, Chochillon C, Matheron J, Datry A, Bouges-Michel C, Bonnal C, Dromer F, Bretagne S (2010). Comparison of antifungal MICs for yeasts obtained using the EUCAST method in a reference laboratory and the Etest in nine different hospital laboratories. Clin Microbiol Infect 16: 863-869.

29. Pfaller MA, Diekema DJ, Sheehan DJ (2006) Interpretive breakpoints for fluconazole and Candida revisited: a blueprint for the future of antifungal susceptibility testing. Clin Microbiol Rev 19: 435-447.

30. Krcmery V, Barnes AJ (2002) Non-albicans Candida spp. causing fungaemia: pathogenicity and antifungal resistance. $\mathrm{J}$ Hosp Infect 50: 243-260.

31. Bouza E, Muñoz P (2008) Epidemiology of candidemia in intensive care units. Int J Antimicrob Agents 32: S87-S91.

32. Rodríguez-Núñez A (2001) Incidence and mortality of proven invasive Candida infections in pediatric intensive care patients. Infect Control Hosp Epidemiol 22: 477- 478.

33. Horn DL, Neofytos D, Anaissie EJ, Fishman JA, Steinbach WJ, Olyaei AJ, Marr KA, Pfaller MA, Chang CH, Webster KM (2009). Epidemiology and outcomes of candidemia in 2019 patients: data from the prospective antifungal therapy alliance registry. Clin Infect Dis 48:1695-1703.

\section{Corresponding author}

Moustafa Abd Elaal Hegazi

Faculty of Medicine in Rabigh, King Abdulaziz University

Jeddah, Saudi Arabia, PO: 80205, Jeddah 21589.

Tel: +966541368683 .

Fax: +966026400592

Mansoura University Children's Hospital,

El-Gomhoria St., PO: 35516. Mansoura, Egypt.

Tel: +201001020941 .

Fax: +20502234092 .

E-mail: mhegazi712003@yahoo.co.uk

Conflict of interests: No conflict of interests is declared. 